\title{
Corrigendum: Dynamic nuclear magnetic resonance field sensing with part-per-trillion resolution
}

Simon Gross, Christoph Barmet, Benjamin E. Dietrich, David O. Brunner, Thomas Schmid \& Klaas P. Pruessmann

Nature Communications 7:13702 doi: 10.1038/ncomms13702 (2016); Published 2 Dec 2016; Updated 27 Feb 2017

The financial support for this Article was not fully acknowledged. The acknowledgements should have included the following: The authors acknowledge Nano-Tera.ch for financial support.

(C) The Author(s) 2017 